\title{
Design and Performance of the Vegetation Canopy Lidar (VCL) Laser Transmitter
}

\section{Barry Coyle}

NASA - GSFC, Code 920.3, Greenbelt, MD 20771

301-614-5859, barry@.comfed.gsfc.nasa.gov

Richard B. Kay, The American University, Washington, DC

Steven J. Lindauer II, NASA-GSFC, Greenbelt, MD 20771

Abstract - The Vegetation Canopy Lidar (VCL) laser is a Nd:YAG Q-switched, diode sidepumped, zig-zag slab design producing $10 \mathrm{~ns}, 15 \mathrm{~mJ}$ pulses at $1064 \mathrm{~nm}$. It employs an unstable resonator as well as a graded reflectivity output coupler with a Gaussian reflectivity profile. In order to conserve power, a conductively cooled design is employed and is designed to operate over a range of $25^{\circ} \mathrm{C}$ without active thermal control. The laser is an oscillator-only design and equipped with an $15 \mathrm{X}$ beam expander to limit the output divergence to less than $60 \mu \mathrm{rad}$. Thermal lensing compensation in the side-pumped slab was performed with different treatments of the $\mathrm{x}$ and $\mathrm{y}$ portions of the $\mathrm{z}$-directed beam. Performance data as a function of temperature are given. 
The Vegetation Canopy Lidar mission (VCL) is a NASA Earth System Science Pathfinder project tasked to measure the volume and structure of the earth's vegetation over a two year period [1]. This free-flying satellite will transmit laser pulses towards the earth's terrain via 3 independent transmitters, each operating at $242 \mathrm{~Hz}$. The reflected pulse waveforms will provide information on the topography and vegetative land-cover for terrestrial ecosystem modeling and climate prediction. Additionally, a reference data set of topographic spot heights and transects for the globe are to be obtained. This will be the first contiguous data set ever obtained for our planet.

The three VCL lasers are to be mounted around the perimeter of the $0.9 \mathrm{~m}$ diameter parabolic receiving mirror of the Multi Beam Laser Altimeter (MBLA) [1]. At the beginning of the mission, each laser is to produce $\sim 15 \mathrm{~mJ}$ of energy per pulse at a repetition rate of $242 \mathrm{~Hz}$. The Earth Scientists who employ the laser return data sets use algorithms on the waveforms to determine characteristics of the land and its vegetation. This technique requires a nearly Gaussian surface footprint with a $1 / \mathrm{e}^{2}$ full-width of approximately $30 \mathrm{~m}$. When one considers the altitude of the spacecraft, the final full-width divergence in the transmitted pulse needs to be about $60 \mu \mathrm{rad}$. Each laser consists of a single box with and an independently sealed electronics compartment and an optics compartment. The optics compartment contains the diode arrays, laser head, temperature and pressure sensors and a kinematically mounted optical bench. The optical bench contains all the cavity optics as well as the external pointing optics and beam expanding telescope (BET). This transmitter produces a $1.0 \mathrm{mrad} 1 / \mathrm{e}^{2}$ full-width beam into the BET, which has a limiting objective diameter of $5 \mathrm{~cm}$. The beam exiting the laser is near $\mathrm{TEM}_{\mathrm{oo}}$ $\left(\mathrm{M}^{2}=1.2\right)$ in order to achieve the above requirements. High efficiency is needed in order to fit 
within the limits of the spacecraft's power budget, therefore a significant effort in this area. The result is a measured optical efficiency of $\sim 14 \%$. When coupled with the state of the art pump electronics and passive radiative cooling, the true "wall-plug" efficiency is $\sim 6 \%$. This efficiency is $67 \%$ greater than that of the best performing laser altimeter transmitter to fly in space to date, the Mars Orbiting Laser Altimeter (MOLA), which just finished mapping Mars [2]. Considering that MOLA was a highly multimode laser, the VCL performance is impressive and demonstrates the state of the art for diode pumped lasers of equivalent beam quality.

\section{Optical Layout}

A schematic of the VCL laser, including the BET, is shown in Fig 1. Its basic plan can be understood by following a sample beam through the system. Starting at the High Reflector (HR) end mirror, the beam travels first through a 0.5 degree Risley pair. [A Risley is a thin wedge shaped disc which rotates independently in a circular mount.] The HR is firmly mounted to the optics bench and the Risleys essentially provide pitch and yaw to/from the HR mirror for cavity alignment. Next, the beam travels through the zig-zag slab, which is pumped vertically by eight 3 bar, 60W/bar, SDL G-package diodes mounted under the slab. The beam exits the zig-zag slab then travels through a negative cylindrical lens to compensate for the strong positive lens created by the pump radiation in the slab. This will be discussed later. After traveling through a hollow, stretched retro reflector (hollow retro) it then encounters a $1 / 2$ plate to rotate the polarization $90^{\circ}$ prior to going through the thin film polarizer. Finally, it travels through the Q-Switch and its $\lambda / 4$ plate, the second 0.5 degree Risley pair, and through an undoped YAG tilt block before arriving at the Graded Reflectivity Mirror (GRM). The tilt block provides beam position while the Risleys adjust pointing. Together, these optics provide cavity alignment and center the beam on the GRM. The portion of the laser cavity radiation transmitted by the GRM travels through the 
second (external) hollow retro and into a single-optic cylindrical beam expanding telescope, used to circularize the laser beam. A $1^{\circ}$ Risley pair is used here to bore site the beam through the BET, consisting of a negative lens group (NLG) and a positive lens group (PLG).

The VCL laser cavity is similar to the high brightness design of Armandillo et.al. created in conjunction with the European Space Agency [3]. The main differences between these two systems are in the laser head design, external beam shaping, and in the details of the unstable resonator including location of elements. Their laser produced $100 \mathrm{~mJ}$ at $100 \mathrm{~Hz}$ and was designed for cloud lidar observations and was also a design for high efficiency.

\section{Unstable Resonator}

Our folded cavity design was dictated by a combination of the size limitations of the launch vehicle and the dimensions of the optic bench set at $30 \mathrm{~cm}$ X $20 \mathrm{~cm}$, approximately. This resulted in folded paths, with a laser geometrical path length of $\mathrm{L}=36 \mathrm{~cm}$. In order to avoid optical damage, it was necessary to keep the beam fluence in the laser resonator below $3 \mathrm{~J} / \mathrm{cm}^{2}$, and preferably below $2 \mathrm{~J} / \mathrm{cm}^{2}$. With a $15 \mathrm{~mJ}$ beam of near $\mathrm{TEM}_{\mathrm{oo}}$ quality, the beam waists needed to be $\sim 1 \mathrm{~mm}$ or greater throughout the resonator to meet this requirement. Standard, stable laser resonators are not well suited to maintaining a large beam waist when the resonator length, $\mathrm{L}$, is short. Unstable resonators, particularly with a GRM, can maintain a large waist and provide some additional benefits.

VCL's unstable resonator was designed in collaboration with the National Optics Institute of Canada, which also produced the GRM output coupler [4]. The most important feature of an unstable resonator is its magnification. Magnification directly affects the dissipative losses (feedback), higher order mode discrimination, alignment sensitivity and the 
GRM spot size relative to the beam waist in the active media $[5,6,7]$. There are a variety of decisions to be made in arriving at a cavity design and its magnification.

The basic cavity design, assuming non-active elements, employs a geometrical length of $36 \mathrm{~cm}$ (diffractive optical length of $30.9 \mathrm{~cm}$ ) with a convex output coupler (GRM) radius of $2.37 \mathrm{~m}$ and a concave $\mathrm{HR}$ reflector of radius $+3.0 \mathrm{~m}$. This provides a total magnification (geometrical plus diffraction) of $\mathbf{M}_{\mathbf{t}}=1.39$. [See Appendix 3, Reference 6] A Gaussian GRM profile was chosen with an intensity reflectivity profile of $R(r)=R_{0} \exp \left[-2\left(r / \omega_{m}\right)^{2}\right]$, where $R_{0}$ is the reflectivity in the center of the mirror and $\omega_{\mathrm{m}}$ is the $1 / \mathrm{e}^{2}$ radius of the deposition profile. In order to sweep out the gain medium, we designed for a beam waist in the Nd:YAG slab $\sim 1.1$ $\mathrm{mm}$. The relationship between $\omega_{i}$ (the beam waist at the output coupler) and the dielectric deposition waist on the mirror is $\omega_{i}=\omega_{m} \cdot\left[\mathrm{M}^{2}-1\right]^{1 / 2}$. Requiring $\omega_{i}=1.1 \mathrm{~mm}$ gives a Gaussian deposition waist of $1.12 \mathrm{~mm}$ for $\mathrm{M} \sim 1.4$.

Much of unstable resonator design can be reasoned-through using the geometrical approximation of Siegman [8]. Using the resonator's $g$ values, $g_{i}=1-L / R_{i}$, the geometrical magnification can be shown to be

$$
M_{g}=G+\left(G^{2}-1\right) \text { where } G=2 g_{1} \cdot g_{2}-1 \text {, }
$$

and is equal to 1.266 for the VCL design resonator. A complete theoretical calculation gives a magnification of 1.389 , which shows the effects of diffraction and the limitation of the geometrical approximation in our case. The mode discrimination between the fundamental mode, $\gamma_{0}$, and higher order modes, $\gamma_{n}$, is 


$$
\gamma_{\mathrm{n}}=\gamma_{\mathrm{o}} /(\mathbf{M})^{2 \mathrm{n}}
$$

and thus, high magnification is desirable. Unfortunately, very high values of magnification give rise to higher losses which can not be overcome in most applications. Substituting a value of 1.39 for the magnification in Eq. 2 indicates we have a factor of $\sim 1.9$ discrimination between the fundamental and next higher mode. The GRM further aids in discrimination against higher order modes by having less reflectivity on the outer portions of the beam as it strikes the mirror.

Feedback for the 1 st order mode has been shown to be $<R>=R_{o} / M^{2}[6,7,8]$. Our requirements were for an effective output coupler feedback of $\sim 0.33$, based on plane wave analysis which included the gain and dissipative losses expected in the physical cavity design $[9,10]$. To achieve an effective reflectivity of $\left\langle R>\sim 0.33\right.$, a Gaussian center reflectivity of $R_{0} \sim$ $63 \%$ was required for $M=1.39$.

Another important consideration is the cavity alignment stability. Krupke has shown that the angular alignment sensitivity, $\mathrm{S}$, can be expressed as [11]

$$
\mathrm{S}=\left[1-\mathrm{g}_{2}\right] /\left[1-\mathrm{g}_{1} \cdot \mathrm{g}_{2}\right]
$$

Smaller values of this parameter are desirable. By definition, unstable resonators have values of $\mathrm{g}_{1} \cdot \mathrm{g}_{2}$ either greater than 1 or less than 0 . For positive branch resonators, which applies in our case, the value of $\mathrm{g}_{1} \cdot \mathrm{g}_{2}$ is not far from unity. It is another design trade-off to arrive at a resonator that has a relatively low $\mathrm{S}$ and a reasonable magnification. We chose a design value of $S \sim 7.4$ for our resonator. This can be compared to a value of $\mathbf{S} \sim 3$ for a very stable resonator with $g_{1} \cdot g_{2} \sim .5$ or to a rather unstable one where $S \geq 50$. This value of $S$ turned out to critical in 
determining our final beam size, intracavity fluence, and ultimately our resulting efficiency and reliability.

\section{Laser Head Design}

The available VCL orbital vehicle could not provide the necessary power or thermal radiative capacity to hold the lasers at a fixed temperature. That meant the laser diode arrays would continually heat up while operating until reaching a predetermined cutoff temperature. Therefore, the VCL mission called for full power operation over land only, to minimize average orbital power consumption and lengthen mission lifetime by reducing the number of laser shots per orbit. A laser transmitter design with the highest "wall-plug" efficiency would provide the slowest heating rate. A thermal radiator panel serves the main purpose of temperature control through a combination of heat pipes and phase change material. (See Section 4)

\section{Pump Module}

The pump module consists of an overall A-shaped structure, a slab clamp, slab heat sink with bonded slab, pump lens and holder, and a series of pump diode arrays. This design is a modification of the one reported on by Hays et al. in 1995 [12]. The module holds the slab from above over the pump lens and diode arrays, which are arranged in a line approximately $8 \mathrm{~cm}$ long. The diodes are eight $\times 3$ bar $60 \mathrm{~W}$ SDL arrays in G packages mounted to a heat sink directly in contact with the bottom of the laser housing. This "pedestal" protrudes through an opening in the optical bench and precisely positions the slab in alignment with the resonator. The diode arrays and cylindrical pump lens create a sheet of pump radiation into the AR coated side of the slab. The opposing slab face is coated with complex combination of $\mathrm{SiO}_{2}$ and multilayer dielectrics to produce both a TIR (total internal reflection) surface and an HR coating for double-passing the $809 \mathrm{~nm}$ pump light. This side is then bonded with thermally conductive 
tape to the slab heat sink. The heat is carried away from the slab heat sink through this surface to the pedestal via a structure that supports these components and thermo-mechanically mounts to the pedestal. This basic design has been employed successfully in multimode designs in the past and symmetric cooling is achieved in the slab. However, we found it necessary to make several changes in order to optimize its performance and qualify it for the rigors of space flight, as are discussed below.

\section{Slab Design}

Our previous work on a similar laboratory laser design showed promise in rethinking the design of the "standard" side pumped, zig-zag Nd:YAG slab [13]. It was learned that significant pump energy was left unused in the slab at the "optimum" slab tip angle proposed by Frantz et al.[14]. They show that a $31^{\circ}$ tip angle can provide the maximum fill factor possible in multimode resonators and amplifiers. However, when high maximum energy extraction in a $\mathrm{TEM}_{00}$ mode is required, as in the VCL transmitter, we found that the tip angle must be reduced. The slab thickness cannot simply be reduced to match our approximately $1.8 \mathrm{~mm}$ diameter beam because we would lose absorbed pump energy. This is because the double pass pump length would only be $3.6 \mathrm{~mm}$. We achieved the best performance for the VCL laser with a slab tip angle of 26.5 deg and thickness of $2.65 \mathrm{~mm}$. Just recently we chose to increase this tip angle $28^{\circ}$ in order to increase our hard aperture in that axis and reduce clipping effects. We were still able to hold our overall efficiency relatively constant. Even though the gain length was reduced by a few $\mathrm{mm}$ due to this larger tip angle, we could now form a slightly wider beam in that axis.

When the calculations are performed with variable tip angles, the number of TIR bounces and the slab thickness are held constant and the slab length is adjusted to compensate. For our estimated beam $1 / \mathrm{e}^{2}$ diameter of $1.8 \mathrm{~mm}$, only $21.6 \%$ of the pumped volume is un-swept by the 
cavity. This is a major improvement over previous designs for the Brewster angled slab and $31^{\circ}$ tip angled slab, where $30 \%$ and $36 \%$ is unswept, respectively. One more advantage of this type of optimized tip angle is a natural single-axis aperture in the zig-zag plane; the smaller the tip angle the smaller this aperture and higher the extraction. One naturally reaches a limit to the tip angle reduction due to the beam thickness overlapping this aperture. We used this inherent aperture to our advantage to control potential off-axis modes.

\section{Pump Lens and its Location}

The diode array output is collected by an undoped YAG, plano-convex, cylinder lens of $1.75 \mathrm{~mm}$ radius and $1.5 \mathrm{~mm}$ center thickness. This produces the energy distribution shown in Fig. 2 which shows the collimated diode array radiation entering the slab from the left. We found that this lens' plano surface position with respect to the diode arrays facets was critical for achieving optimal performance in the laser cavity. The pump beam must not be too tightly focused or the region of high inversion density will create too strong a thermal lens. On the other hand, this region must somewhat under-fill the laser beam for good extraction efficiency.

An optimum lens/slab design combination was found through several iterations of modeling and experiment. We eventually produced a very uniform pump beam, about $1.5 \mathrm{~mm}$ thick. This is seen in the end view of spontaneous radiation emanating from the slab shown in Fig 3 .

\section{Thermal Lens}

The $\sim 1.5 \mathrm{~mm}$ wide pump beam creates a thermal lens in the slab. Isotherms from thermal modeling of the pumped slab are shown in the cross sectional view of Fig. 4. In this figure, $\sim 23$ $\mathrm{W}$ of average power is incident on the slab in a centered $1.5 \mathrm{~mm} \times 85 \mathrm{~mm}$ region. The pump beam travels through the slab in the $\mathrm{Y}$ axis and its pump face, the $\mathrm{Z}$ axis, is about $95 \mathrm{~mm}$ long. 
The opposing HR slab face is attached to the heat sink and held at a reference temperature of $0^{\circ} \mathrm{C}$. [The width of the slab, along the $\mathrm{X}$ axis, is $5 \mathrm{~mm}$ and its height is $2.65 \mathrm{~mm}$.] The isotherms show that an electromagnetic wave, $E_{Y}$, will experience a different thermal gradient (and thus optical length) on center $(x=0)$ than to either side $(x= \pm a)$. If the isotherms develop too to each other, then the strength of the thermal lens becomes too strong producing a near stable cavity in that axis from which optical damage is more likely to occur. A reduction in the thermal lens strength was obtained by creating a "stepped" heat sink with a $2 \mathrm{~mm}$ wide ridge down the length for the slab's bonding surface. This is seen in Fig 5. By increasing thermal path's resistance toward the slab edges, their steady state temperatures are elevated. The slab's overall thermal gradients are reduced which produces a weaker positive lens.

Thermally conductive, double sided bonding tape, typically used for semiconductor bonding to heat sinks and circuit boards, is used to adhere the HR slab face to the heat sink. This tape has a fine weave of aluminum wire imbedded in a bonding agent. A pair of tape strips is applied down the heat sink length on each side of the step, then a single tape strip is applied over the entire width of the heat sink where the slab is then applied. The step is carefully machined to 0.009 " height $(+0.000 /-0.001)$, the compressed tape thickness, in order to provide a smooth bonding surface for the slab with minimum air pockets. The heat sink is made of a tungsten/copper material, appropriately called Thermkon by its manufacturer, which closely matches the thermal expansion coefficient of Nd:YAG, thereby theoretically reducing any mechanical strain of deformation on the slab over temperature.

\section{Thermal Lens Effects on Cavity Operation}

The steady state thermal lens, measured with a $1.06 \mu \mathrm{m}$ test beam, is found to be weaker when the laser is operating, as opposed to measuring the operating head assembly out of the cavity. 
When lasing, stimulated-emission-cooling of the inversion, also known as radiative cooling, changes the stored heat distribution in the slab and measurably reduces the lens. Due to this effect, direct thermal lens measurements, which can only be done without a supporting laser cavity, proved to be significantly stronger than when the laser is in steady state operation. When this laser is operating at about $16 \mathrm{~mJ}$ measured prior to the telescope, a positive, "cylindrical" thermal lens of focal length $f \approx+82 \mathrm{~cm}$ is created in the slab in the $X$ axis. Additionally, the slab creates a weak negative lens of focal length of -7.8 meters in the $Y$ direction. To counter the weak negative lens, the radius of the HR was changed from the original (empty cavity) design of $R=+3 \mathrm{~m}$ to $\mathrm{R}=+2 \mathrm{~m}$. This compensated the $\mathrm{Y}$ axis nearly to the original design parameters, as determined by active beam waist measurements at both the GRM and the HR, and comparison with Paraxia modeling [16]. For the $X$ axis, the combination of the $R=2 m$ HR, plus a $f=-70$ $\mathrm{cm}$ cylindrical lens, completed the compensation. In both cases, beam waist measurements were compared to Paraxia modeling of the cavity, which included a thick lens to approximate the slab's thermal lens. In this way, the operating thermal lens focal lengths were accurately deduced.

\section{Conductive cooling}

Each laser is cooled via a phase change module integrated with a radiator panel. The basic thermal configuration is an aluminum honeycomb radiator with four embedded heat pipes and a phase change module mounted between the radiator and laser transmitter. Each of the radiators is a $39 \times 89 \mathrm{~cm}$ rectangle, and together they serve a second purpose as optical baffles for the primary telescope mirror. Survival heaters for each laser are controlled by the electronics using set points uploaded from the ground. 
To conserve power and laser shots (lifetime), the lasers operate at minimum average power (10 Hz) while over the ocean. This is more of a keep-alive operation to keep the lasers warm such that rapid peak power operation can be achieved when land coverage arrives. While over land, the two coolest lasers are chosen by the control electronics to run at maximum power $(242 \mathrm{~Hz})$ while the third laser stays in minimum power mode.

The laser pump source is a set of eight 3-bar diode array stacks, assembled side by side on the pedestal such the collective pump source is about $1 \times 80 \mathrm{~mm}$. Any single diode bar has typical FWHM emission spectrum of $\sim 3-4 \mathrm{~nm}$. However, a set of 24 bars has a wider spectrum of about $6 \mathrm{~nm}$ FWHM. This is partly due to each array being at a somewhat different temperature due to its location along the heat sink. Those bars in the center will be hotter than those at the ends. While this spectrum does not change in width over temperature, the center wavelength will shift at a rate of $0.3 \mathrm{~nm} /{ }^{\circ} \mathrm{C}$. The absorption spectrum of $\mathrm{Nd}$ :YAG has a sharp peak at $809 \mathrm{~nm}$, approximately $0.5 \mathrm{~nm}$ FWHM, which does not move appreciably with temperature. The combination of the $\sim 6 \mathrm{~nm}$ diode spectrum and the temperature tuning of these diodes allows the laser to operate over a $25^{\circ} \mathrm{C}$ temperature range (Fig. 6). When the diodes are at $12^{\circ} \mathrm{C}$, the peak wavelength is $805 \mathrm{~nm}$, but enough of the diodes' emission overlaps the absorption line so that the laser operates at the minimum acceptable energy, $\sim 10 \mathrm{~mJ}$. A similar situation exists at $32^{\circ} \mathrm{C}$ (emission peak now at $811 \mathrm{~nm}$ ), but it is the opposite wing of the emission spectrum that overlaps the absorption line.

\section{Performance}

Peak output energy of $\sim 15 \mathrm{~mJ}$ is being produced at a temperature of $\sim 22^{\circ} \mathrm{C}$ with excellent divergence figures and $\mathrm{M}^{2}$ values. Figure 7 presents the change in energy with temperature and Figure 8 shows the divergences exiting the transmitter. The laser is able to produce meaningful 
energy between $10-35^{\circ} \mathrm{C}$ with pulsewidths of $\sim 10 \mathrm{~ns}$. The optical efficiency of the complete transmitter at the design output of $15 \mathrm{~mJ}$ is $13.9 \%$. This is when driving the pump diodes at 59 W with an $85 \mu$ s pulse for a $110 \mathrm{~mJ}$ input pulse energy. After the GRM output coupler, there are still 24 optical surfaces until the pulse leaves the spacecraft. All these external optics subtract another $\sim 13 \%$ from the laser oscillator output. The laser itself is outputting about $17.4 \mathrm{~mJ}$ and has an optical efficiency of $>15 \%$.

We have found the efficiency of SDL diodes to be within $\sim 2 \%$ of that stated by SDL on the device data sheets. A custom developed DC to DC converter and diode drive unit has demonstrated $88 \%$ overall efficiency [16]. With this converter the overall efficiency of the transmitter is $\sim 6.4 \%$ at $15 \mathrm{~mJ}$ output energy.

\section{Beam Shape and Quality}

Good beam quality is important for LIDAR laser transmitters designed for precise terrain mapping and for measuring vegetation canopies. While our design is currently not able to produce the same beam quality in both axes ( $\mathrm{X}$ and $\mathrm{Y}$ ), we were able to obtain a stable, high quality, far field pattern, with low divergences.

Figure 9 presents a typical far field pattern at the focus of an $f=3.43 \mathrm{~m}$ spherical lens with the laser operating at $\sim 15 \mathrm{~mJ}$ output. The profile plots show the small differences in beam shape for the two axes. The vertical ( $\mathrm{Y})$ axis is associated with the strong thermal lens in the slab which was compensated in this case with an $\mathrm{f}=-75 \mathrm{~cm}$ cylindrical lens. At $15 \mathrm{~mJ}$, the ${ }^{1} / \mathrm{e}^{2}$ beam diameters in the far field are $D_{x}=208 \mu \mathrm{m}$ and $D_{y}=199 \mu \mathrm{m}$, yielding full angle divergences of $\theta_{X} \sim 60.7 \mu \mathrm{rad}$ and $\theta_{\mathrm{Y}} \sim 58 \mu \mathrm{rad}$. The beam shape images exiting the telescope

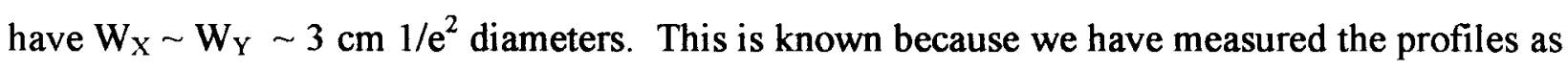
they exit the GRM and adjusted the $\mathrm{X}$ and $\mathrm{Y}$ axis telescope magnifications to produce this result. 
The overall beam quality is estimated to be $\theta_{\mathrm{x}} \cdot \mathrm{W}_{\mathrm{X}} \sim 182 \mathrm{~mm}-\mathrm{mrad}$ and $\theta_{\mathrm{Y}} \cdot \mathrm{W}_{\mathrm{Y}} \sim 174 \mathrm{~mm}-\mathrm{mrad}$ giving values of $\left[\mathrm{M}_{\mathrm{x}}\right]^{2} \sim 1.35$ and $\left[\mathrm{M}_{\mathrm{y}}\right]^{2} \sim 1.28$.

\title{
Conclusions
}

The combination of a diode pumped, zig-zag slab laser head and an unstable resonator cavity has proven to be a success. The output powers and efficiencies have met or exceeded specifications. The unstable resonator's use of a GRM output coupler with an optimally designed slab produces clean, almost diffraction limited far field beams. This cavity is in fact reasonably stable, and has passed vibration tests. These tests put the laser through a spectrum of vibration frequencies similar to, but $50 \%$ greater in acceleration than, what would occur in a launch. To date the pump laser diode arrays are holding up with time, but are still considered to be one of our higher risk components. No discernable output drop has been seen in a $50 \mathrm{hr}$ performance test in which the laser was repeatedly cycled in temperature.

\begin{abstract}
Biography
D. Barry Coyle received the B.S. degree in physics from The College of Charleston, Charleston, SC, in 1986 and the M.S. and Ph.D. degrees in physics from The American University, Washington, DC, in 1986 and 1992, respectively.

He spent the summer of 1988 at Oak Ridge National labs in Tennessee working for the Analytical Sciences section. He was a National Research Council Post-doctoral Fellow for 2 years, working at NASA's Goddard space flight center (GSFC) in the Photonics Branch developing diode-pumped solid state laser transmitters for aircraft-based altimetry. Through a grant with the American university, he continued work at GSFC developing new airborne laser transmitter technology and eventual space based laser development. He is now a NASA employee in the Geoscience Technology Branch where he builds spaceborne laser transmitters. His current research interests include high power diode pumping technology, new laser material applications, and assorted laser-based remote sensing instrument applications and development.
\end{abstract}

Richard B. Kay received the B.S. degree in mathematics and physics from Drury College, Springfield, MO, in 1959, the M.S. degree in physics from the University of Arkansas in 1962, and the Ph.D. degree in physics from the University of Arkansas, Fanetteville, in 1966.

He spent two years in Laser R\&D at McDonnell Aircraft Corporation, St. Louis, MO, then returned to the University of Arkansas for his Ph.D. work. He was a Post-Doctoral Fellow at The University of Florida before coming to The American University, Washington, DC, where he has been a Professor of Physics for 30 years, including a stint as Chairman between 1976-1986. Much of his early research was in atomic collision physics which led into laser-induced multiphoton studies, with concurrent development of specialized solid-state lasers. Since 1986, he has been involved in developing lasers and laser systems in cooperation with NASA's Goddard Space Flight C'enter, with American University and NASA colleagues, and his graduate students. C'urrent work 
involves development of diode-pumped solid-state Nd:YAG oscillators and amplifiers for flight and space applications.

Steven J Lindauer II received the B.S. degree in Physics and the B.S. degree in Mathematics from the College of Charleston, Charleston, SC, in 1996 and 1997, respectively, and the M.S. degree in Optics from the School of Optics / CREOL at the University of Central Florida, Orlando, FL, in 2000.

Before entering graduate school, he worked for The American University at NASA's Goddard Space Flight Center on novel laser amplifier designs and the Laser Vegetation Imaging System's (LVIS) third version laser system. His current research interests include laser-based remote sensing instrument applications and development; laboratory software development for instrumentation control, data automation, and remote access; and optical component and system design. 


\section{References}

1. Information about these NASA projects is most readily available on the internet: http://essp.gsfc.nasa.gov/vcl/and http://www.gsfc.nasa.gov/division/VCLhome/VCLInst.html

2. R. S. Afzal, "Mars Observer Laser Altimeter - Laser Transmitter," App. Opt. 33, 3184-88, (1994).

3. E. Armandillo, C. Norrie, A. Cosentino, P. Laporta, P. Wazen and P. Maine, "Diode-Pumped High Efficiency High-Brightness Q-Switched Nd:YAG Slab Laser," Opt. Let. 22, 1168-70 (1997).

4. National Optics Institute, (NOI), 369 Franquet, Saint-Foy, Quebec, Canada, Glp-4N8. M. Morin, technical consultant (morin(a)ino.qc.ca). (See also reference 6.)

5. De Silvestri, S, Laporta P, Magni, M. and Svelto, O., "Solid-State Laser Unstable Resonators with Tapered Reflectivity Mirrors: The Super-Gaussian Approach”, IEEE J QE 24, 1172-1177 (1988).

6. Moran, M., "Graded Reflectivity Mirror Unstable Laser Resonators", Opt. and QE 29, 819-866 (1997).

7. Mudge, D, Veitch, P. J., Jasper, M., Ottaway, D., and Hamilton, M. W., "High-Power Diode-Laser-Pumper CW Solid-State Lasers Using Stable-Unstable Resonators", IEEE J. Sel. Top. QE 3, 19-25, (1997).

8. Siegman, A. E., "Unistable Optical Resonators for Laser Applications", Proc. IEEE 53, 277-287, (1965). See also Sooy, IELE JQE 5, 575 (1969).

9. Degnan, J. J., "Theroy of the Optimally Coupled Q-switched Laser", IEEE JQE 25, 214 -220 (1989).

10. D. Barry Coyle, David V. Guerra and Richard B. Kay, "An Interactive Numerical Model of Diode-Pumped, Q-Switched/Cavity Dumped Lasers", J. Appl Phys. 28, 452 - 62 (1995).

11. W. F. Krupke and W. R. Sooy, "Properties of an Unstable Confocal Resonator $\mathrm{CO}_{2}$ Laser System", IEEE JQE 5, $575-86,(1969)$

12. Hays, A.D., Witt, G., Martin, N., DiBiase, D., and Burnham, R. "Ultra-Compact Diode-Pumped Solid-State Lasers", Proceedings of the SPIE, 2380, 88-94 (1995)

13. D. Barry Coyle, Steven J. Lindauer II and Richard B. Kay, "Development of a Medium Repetition Rate (10 Hz$500 \mathrm{~Hz}$ ) Diode Pumped Laser Transmitter for Airborne Scanning Altimetry", Nineteenth International Laser Radar Conference (19-ILRC) July 1998, Annapolis MD.

14. J. M. Eggleston, L. M. Frantz and H. Injeyan, "Derivation of the Frantz-Nodvik Equation for Zig-Zag Optical Paath, Slab Geometry Laser Amplifiers", IEEE JQE 25, 1855-1862, (1989)

15. Paraxia is a general laser beam propagation and laser resonator analysis program. See www.sciopt.com.

16. Alan Lukemeyer, Private Communication. 


\section{Figure Captions}

Figure 1. VCL laser schematic

Figure 2. Calculated pump distribution

Figure 3. Spontaneous emission from laser slab

Figure 4. Thermal model of laser slab

Figure 5. Thermal model with stepped heat sink

Figure 6. Diode Pump Overlap with Nd:YAG absorption peak (D) at $12^{\circ} \mathrm{C}(\mathrm{A}), 22^{\circ} \mathrm{C}(\mathrm{B})$, and $32^{\circ}(\mathrm{C})$

Figure 7. Energy vs. Temperature

Figure 8. $\mathrm{X}$ and $\mathrm{Y}$ Divergences vs. Temperature

Figure 9. Far field pattern 


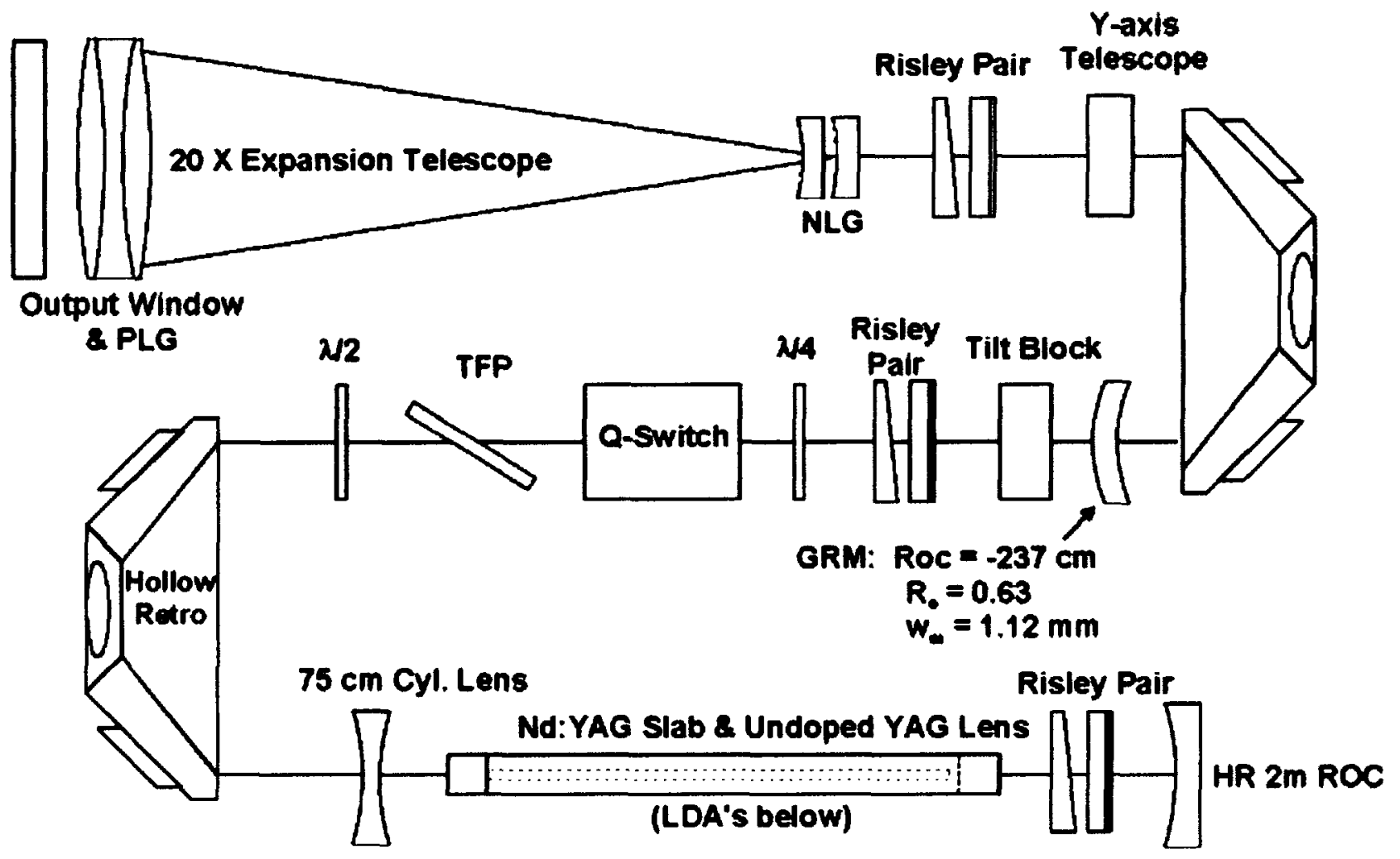

Figure 1. 


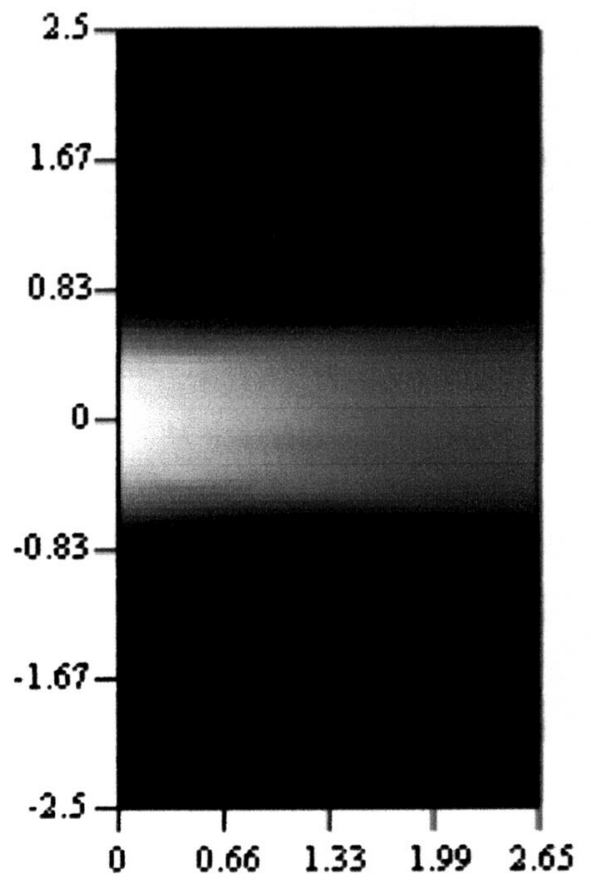

Figure 2. 


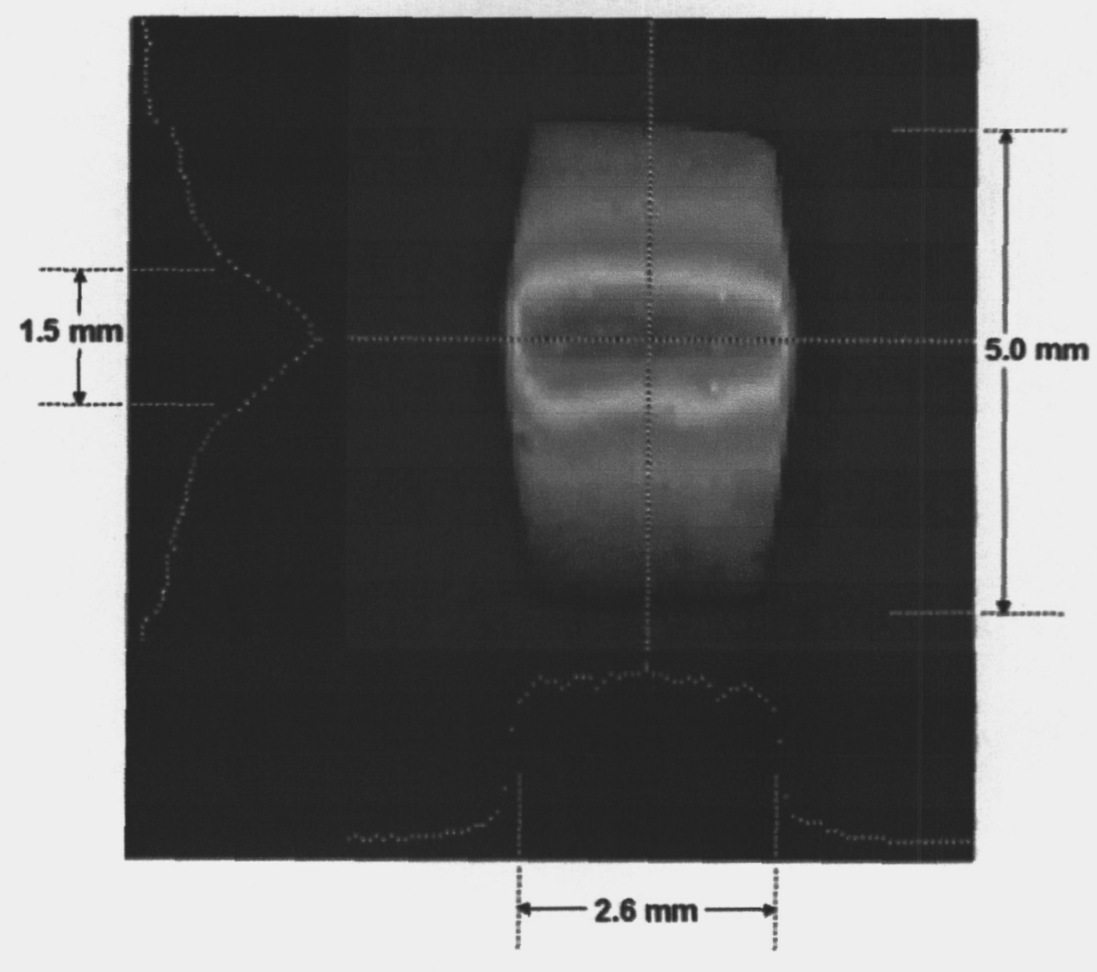

Figure 3 


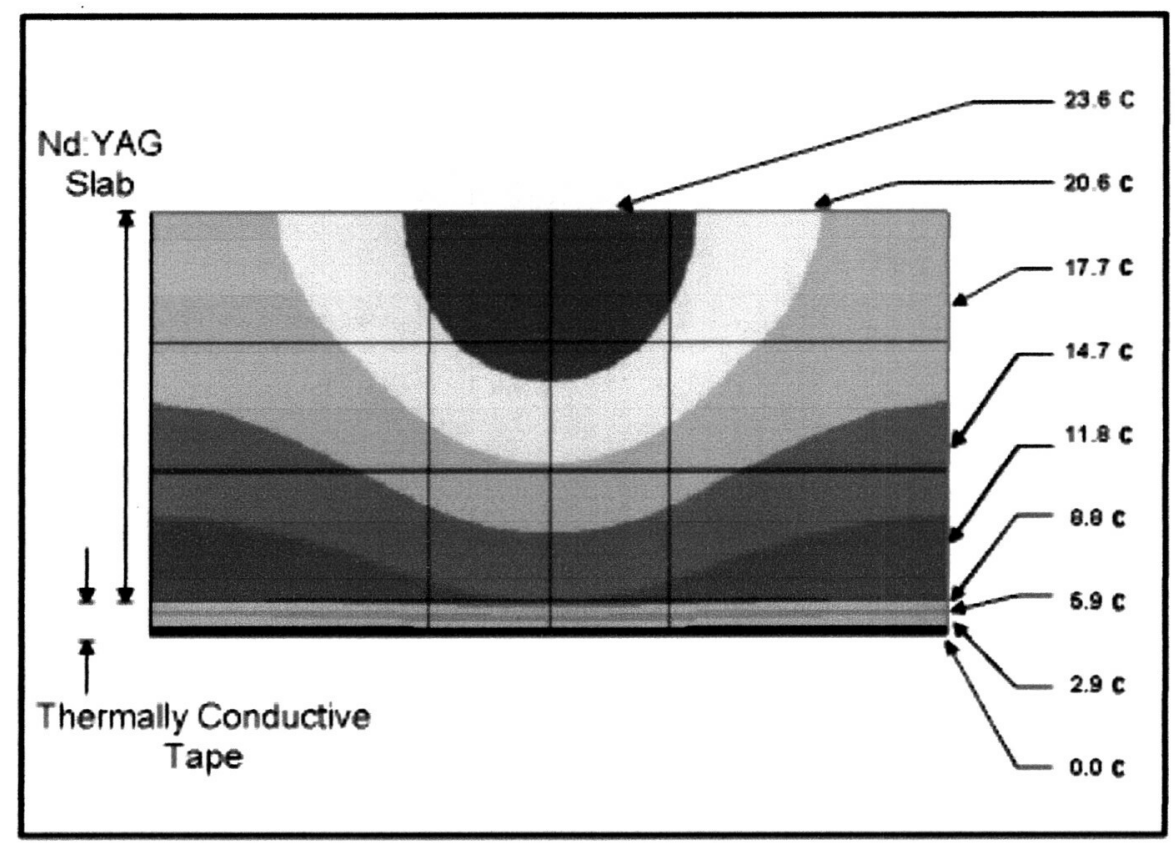

Figure 4 


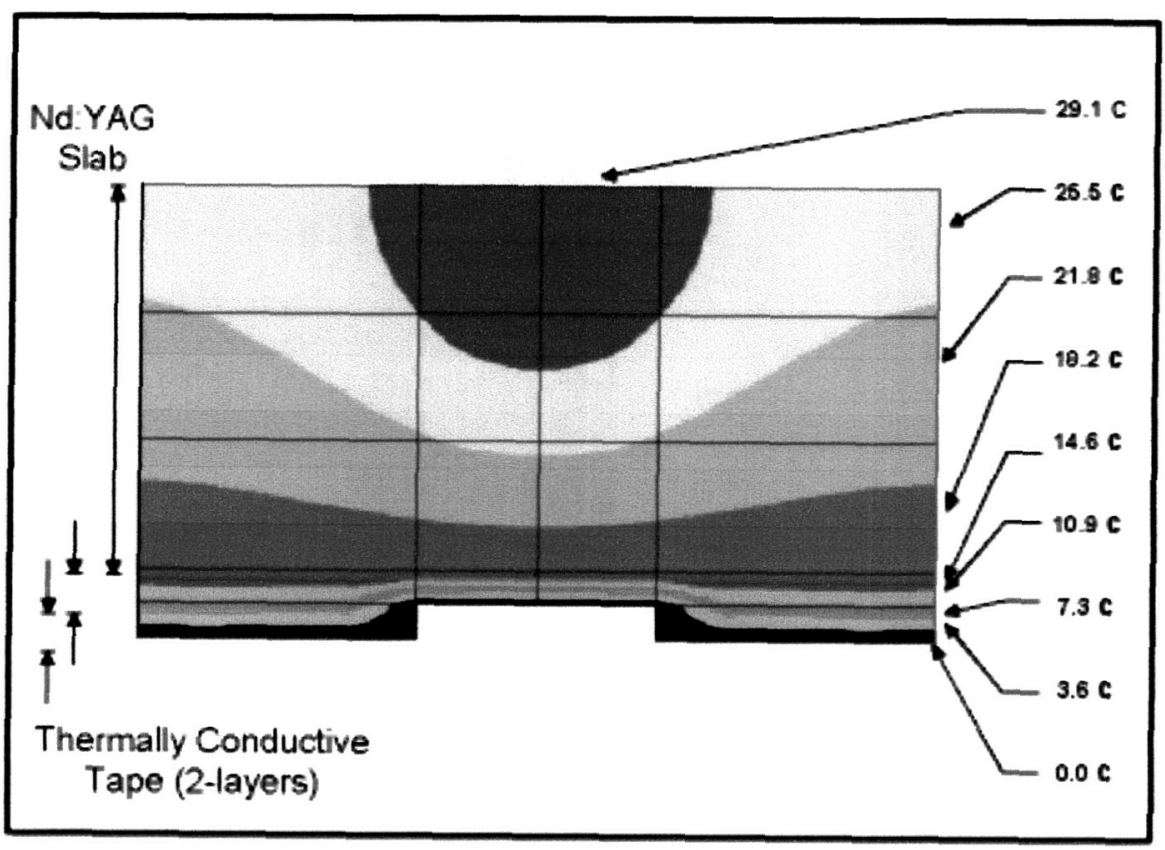

Figure 5 


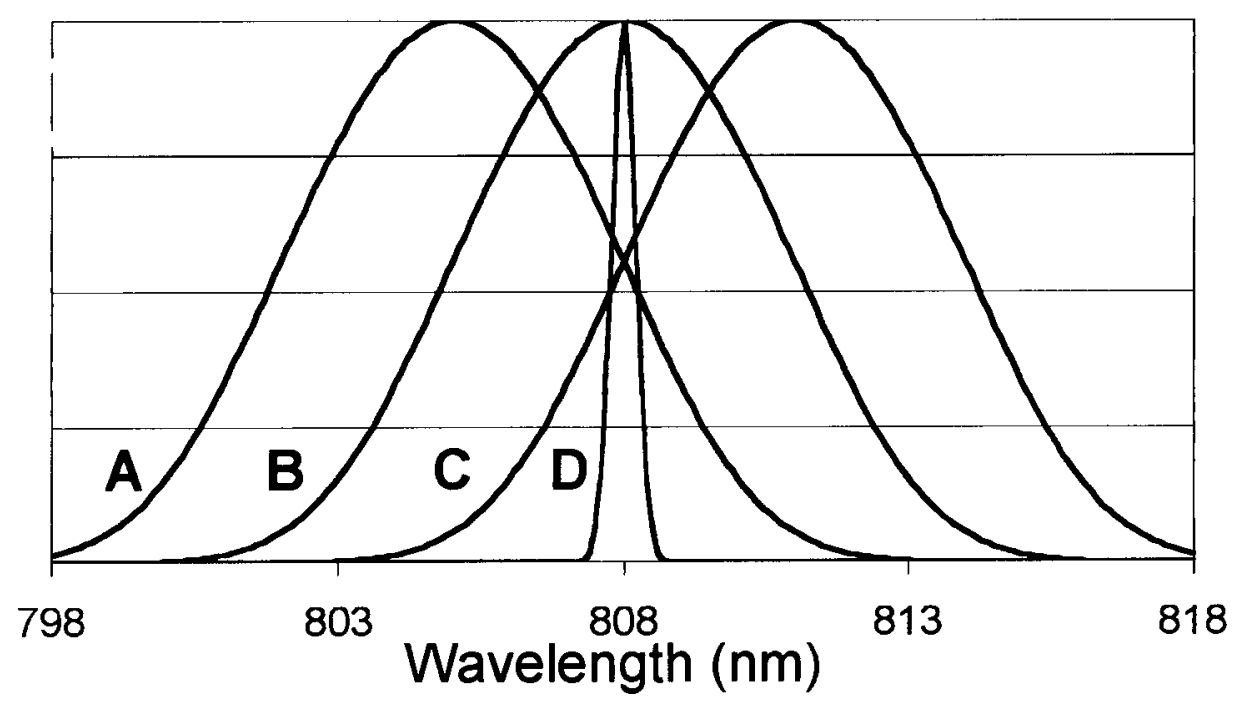

Figure 6 


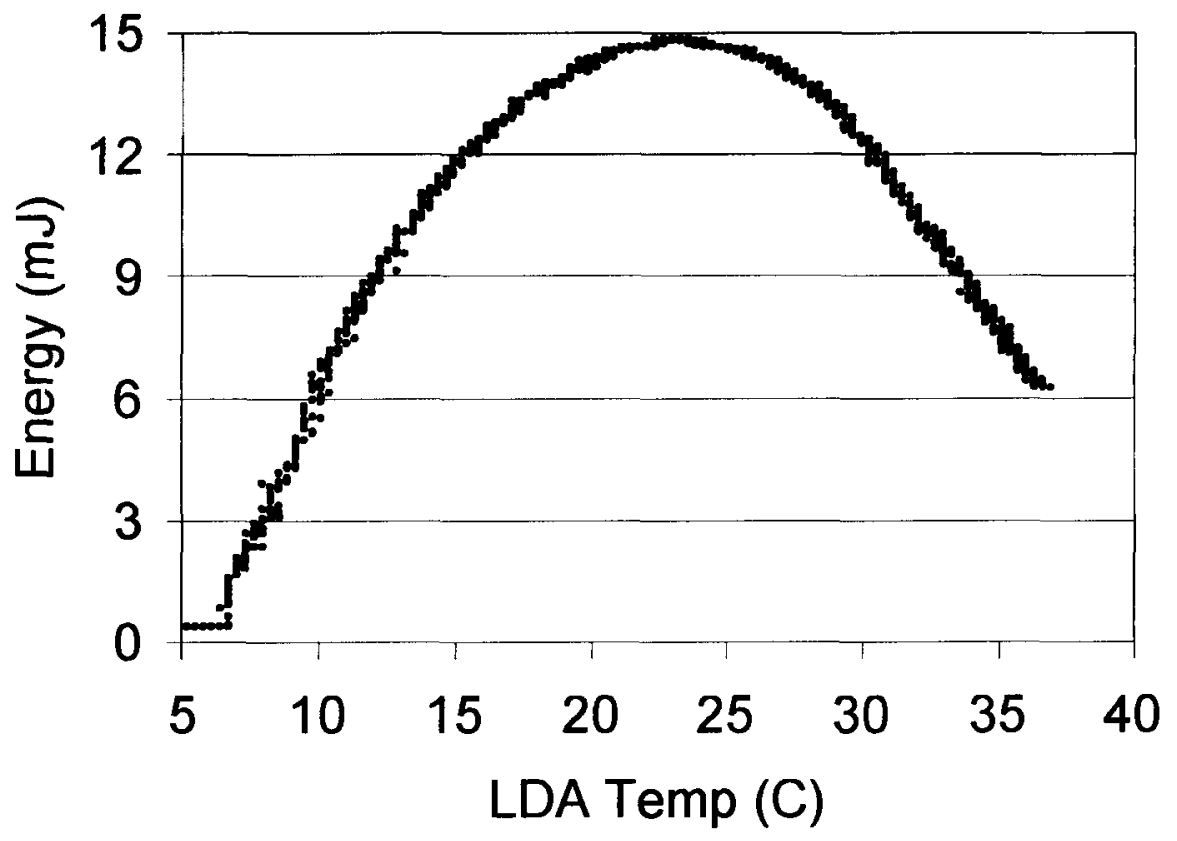

Figure 7 


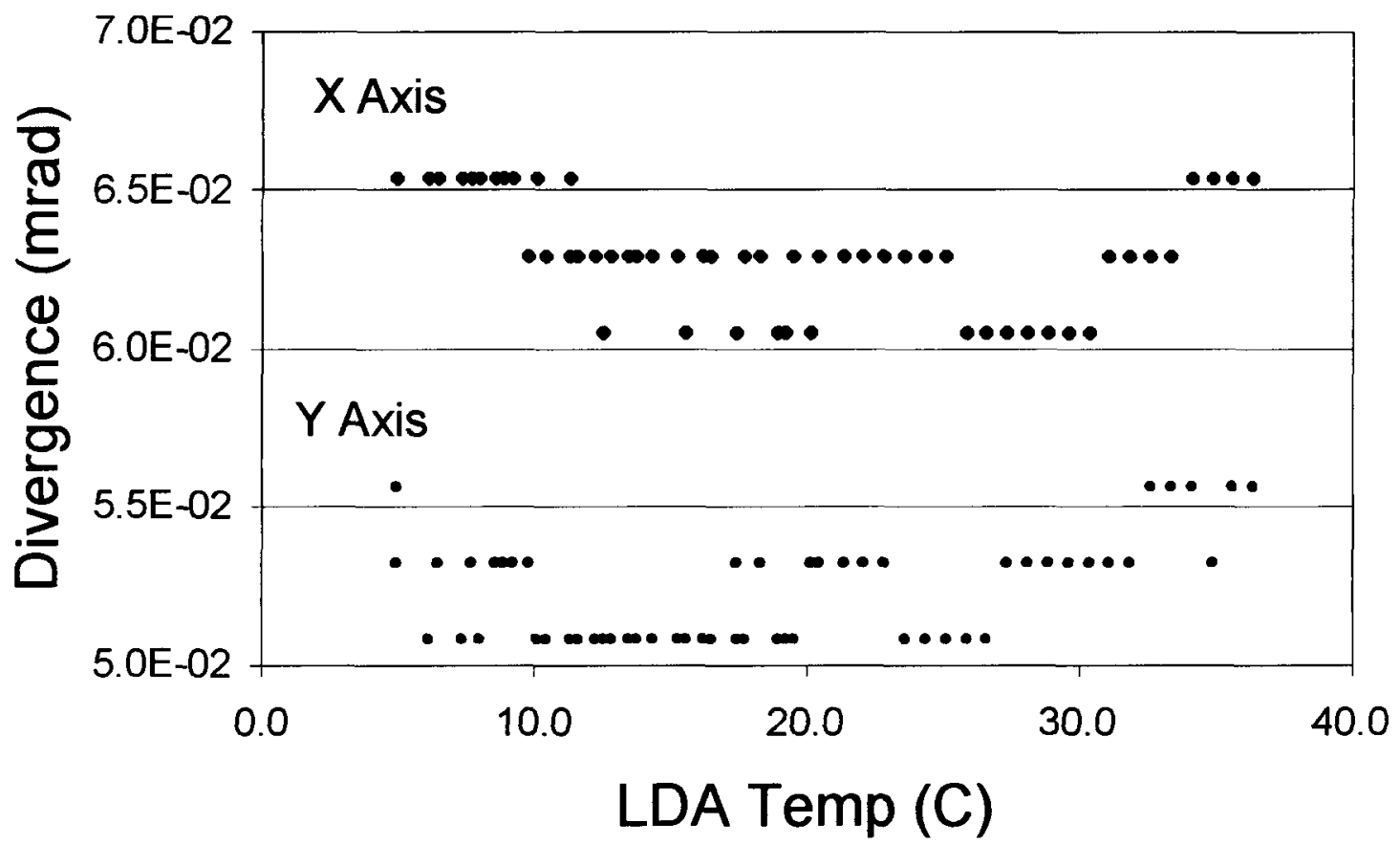

Figure 8 


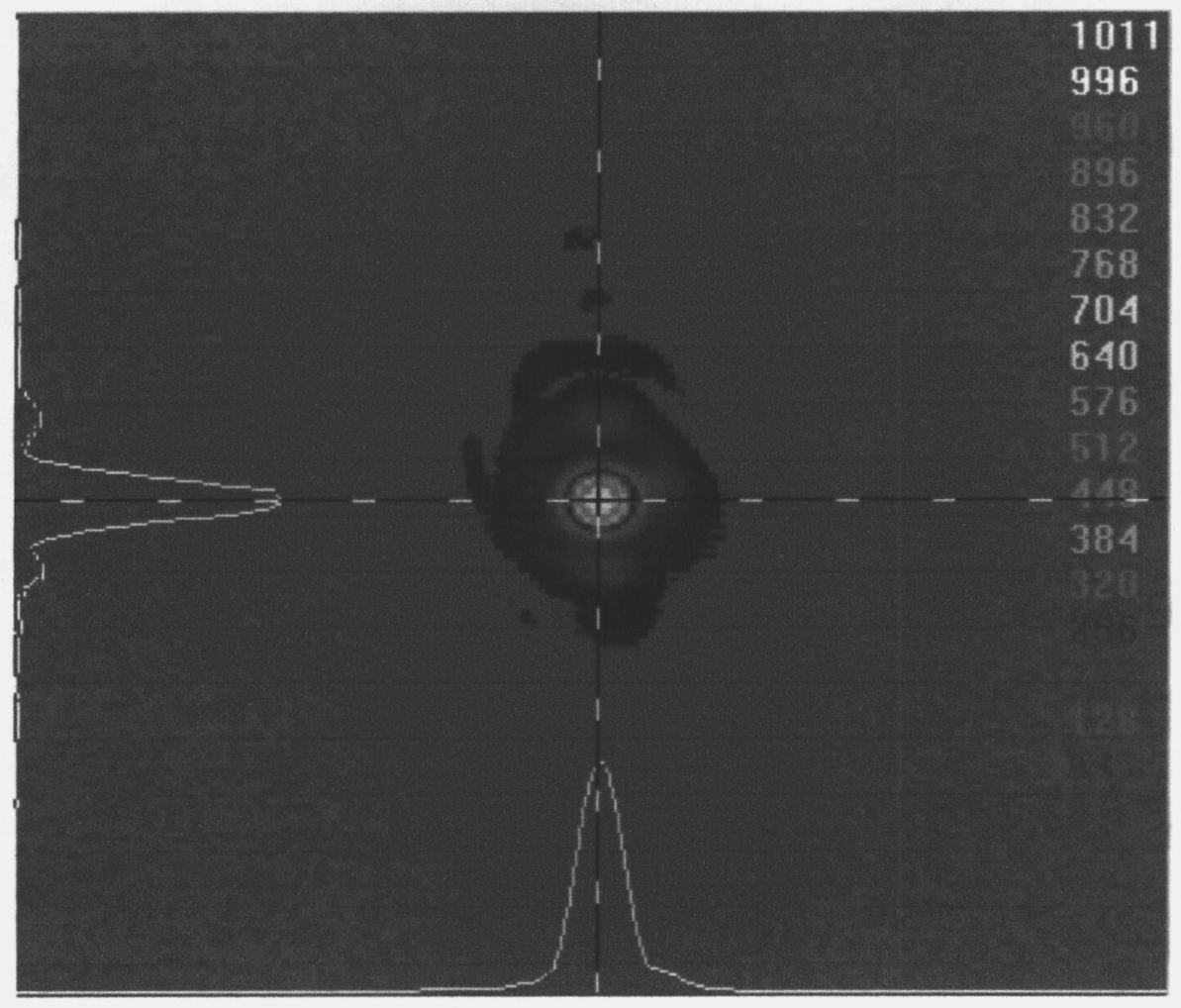

Figure 9 